\title{
Self-assembled $\mathrm{ZnO}$ quantum dots with tunable optical properties
}

\section{$\operatorname{AUTHOR}(\mathrm{S})$ :}

Lu, JG; Ye, ZZ; Zhang, YZ; Liang, QL; Fujita, S; Wang, ZL

\section{CITATION:}

Lu, JG ...[et al]. Self-assembled ZnO quantum dots with tunable optical properties. APPLIED PHYSICS LETTERS 2006, 89(2): 023122.

\section{ISSUE DATE:}

2006-07-10

URL:

http://hdl.handle.net/2433/39682

\section{RIGHT:}

Copyright 2006 American Institute of Physics. This article may be downloaded for personal use only. Any other use requires prior permission of the author and the American Institute of Physics. 


\title{
Self-assembled $\mathrm{ZnO}$ quantum dots with tunable optical properties
}

\author{
J. G. Lu, Z. Z. Ye, ${ }^{\text {a) }}$ and Y. Z. Zhang \\ State Key Laboratory of Silicon Materials, Zhejiang University, Hangzhou 310027, \\ People's Republic of China \\ Q. L. Liang \\ Quality Engineering Division, Semiconductor Manufacturing International Corp., Shanghai 201203, \\ People's Republic of China \\ Sz. Fujita \\ International Innovation Center, Kyoto University, Katsura, Nishikyo-ku, Kyoto 615-8520, Japan
}

Z. L. Wang

School of Materials Science and Engineering, Georgia Institute of Technology, Atlanta, Georgia 30332-0245

(Received 31 March 2006; accepted 24 May 2006; published online 14 July 2006)

\begin{abstract}
Self-assembled $\mathrm{ZnO}$ quantum dots (QDs) were achieved by a vapor phase transport process. $\mathrm{ZnO}$ nanodots were naturally formed on solid substrates in the Volmer-Weber growth mode. Size control of nanodots could be readily realized by varying the growth time. The as-prepared $\mathrm{ZnO}$ QDs are of high quality and very stable after formation. The blueshift of band gap energies derived from quantum confinement effects was confirmed by optical absorption spectra. Photoluminescence spectra revealed the tunable behavior of ultraviolet luminescence due to exciton localization. The realization of size-tuned color from $\mathrm{ZnO}$ QDs makes them more promising for practical applications. (C) 2006 American Institute of Physics. [DOI: 10.1063/1.2221892]
\end{abstract}

Semiconductor quantum dots (QDs) are known for unique behaviors, having the size-tunable atomiclike properties arising from quantum confinement in the nanometer scale. II-VI semiconductor QDs such as CdSe and ZnSe, ${ }^{1-3}$ as compared with IV and III-V materials, ${ }^{4-6}$ have attracted more attention due to the higher exciton binding energies and stronger exciton-photon interactions in these materials. Unfortunately, the band gap of bulk CdSe is $1.74 \mathrm{eV}$, which is difficult to be tuned to ultraviolet (UV) range, and moreover it is toxic and cannot be easily used for human medical applications; ZnSe can be used in the UV-blue region, but its related devices are heavily degraded by the resulting defects. $\mathrm{ZnO}$, having a wide direct band gap of $3.37 \mathrm{eV}$ at room temperature, is a promising candidate for short-wavelength devices. Due to the large exciton binding energy of $60 \mathrm{meV}$, $\mathrm{ZnO}$ is known to be the brightest emitter of available widegap semiconductors. ${ }^{7}$ In addition, $\mathrm{ZnO}$ has other significant attributes, such as high defect resistance, long-term stability, environmental friendliness, biosafeness and biocompatibility, and low material cost. In these regards, $\mathrm{ZnO}$ QDs gain the advantage over $\mathrm{CdSe}$ and $\mathrm{ZnSe}$ for practical applications. ${ }^{8}$

$\mathrm{ZnO}$ QDs or nanoparticles are prepared mainly by wet preparation methods. $^{9-12}$ These techniques require large amounts of solvents, multiple process steps, minute control on composition, as well as long processing times. The controlled growth is very difficult. Recently, the selective growth of $\mathrm{ZnO}$ QDs has been reported by a metal-organic chemical vapor deposition (MOCVD) technique on $\mathrm{SiO}_{2} / \mathrm{Si}$ substrates. ${ }^{33}$ More recently, we have developed a vapor phase transport (VPT) growth process to produce ZnO QDs directly on $\mathrm{Si}$ and such substrates. ${ }^{14}$ The realization of $\mathrm{ZnO}$ QDs on conventional solid substrates gives us an opportunity to gain a fundamental understanding of this nanostructure. In

\footnotetext{
${ }^{a)}$ Electronic mail: yezz@cmsce.zju.edu.cn
}

this letter, we demonstrate that the VPT process allows the creation of self-assembly of $\mathrm{ZnO}$ QDs in the Volmer-Weber growth mode, with controllable nanodot sizes. The sizetuned optical properties were firmly identified by optical absorption and photoluminescence (PL) spectra.

ZnO QDs were synthesized by a VPT growth process under controlled conditions. Detailed growth process could be found elsewhere. ${ }^{14}$ Briefly, ZnO QDs were synthesized by thermally evaporating zinc acetate powders at $500{ }^{\circ} \mathrm{C}$ with a $20 \% \mathrm{O}_{2} / \mathrm{Ar}$ mixture gas flow. The deposition time varied from 2 to $4 \mathrm{~min}$. The products were collected on a substrate placed at a relatively low temperature for property examinations. The morphologies and structures of the products were investigated by a FEI Sirion 200 FEG field-emission scanning electron microscope (FE-SEM), a Philips CM200 transmission electron microscope (TEM), and a Bede D1 X-ray diffraction (XRD) system. The chemical composition was analyzed using an energy dispersive $\mathrm{X}$-ray spectroscopy (EDX) attached to the SEM. PL measurements were performed at room temperature by using a $\mathrm{He}-\mathrm{Cd}$ laser $(325 \mathrm{~nm})$ as the excitation source. Absorption was measured with a Lambda $20 \mathrm{UV}$-visible spectrometer.

Figure 1 illustrates the SEM micrographs of products collected on Si substrates with different growth durations. The images clearly identify the appearance of $\mathrm{ZnO}$ QDs, and essentially no obvious aggregation can be found. The formation of wurtzite $\mathrm{ZnO}$ nanocrystals was confirmed by EDX and XRD observations (not shown here), which are similar to those illustrated in our previous report. ${ }^{14}$ Size control of $\mathrm{ZnO}$ QDs can be achieved simply by varying the growth time. The observed average nanodot diameters increase approximately from 10 to 15 to $21 \mathrm{~nm}$ as the growth time increases from 2 to 3 to $4 \mathrm{~min}$, with their nanodot densities about on the same order of $10^{11} \mathrm{~cm}^{-2}$. Almost the same results have been obtained on sapphire substrates. 

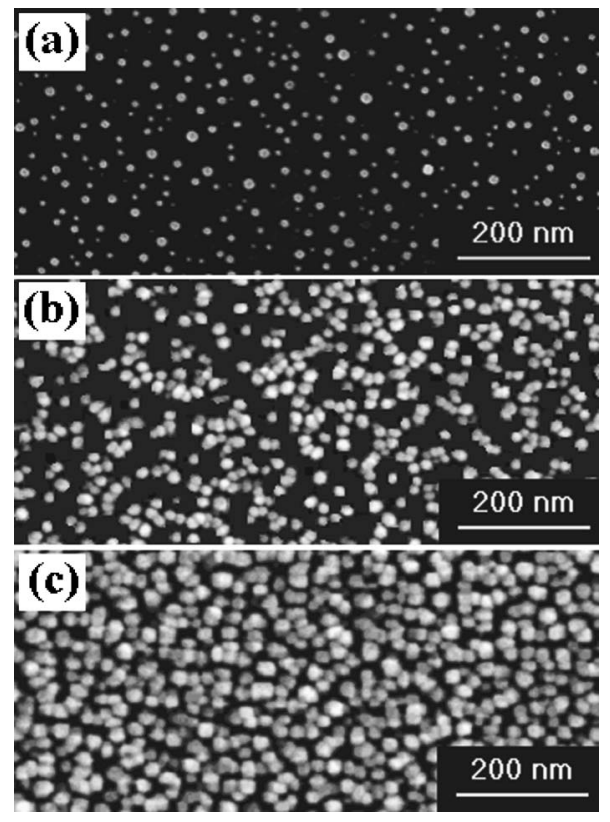

FIG. 1. SEM images of as-prepared $\mathrm{ZnO}$ QDs with different growth times of (a) 2, (b) 3, and (c) 4 min.

It should be noted that the nanodot diameter observed in SEM is within the base area of the dot, and so usually overestimated as compared with the most probable value obtained from analysis of the TEM images. Thus, to illustrate the morphology of $\mathrm{ZnO}$ QDs precisely, TEM measurements were performed on samples. Figure 2 shows the plan-view and cross-sectional TEM images of $\mathrm{ZnO}$ QDs deposited for 2 min as an example. The TEM observations, together with the SEM images shown above, serve to firmly establish the naturally direct growth of $\mathrm{ZnO}$ QDs on solid substrates. We demonstrate that $\mathrm{ZnO}$ QDs could be formed in the VolmerWeber growth mode. The Volmer-Weber QDs or islands have

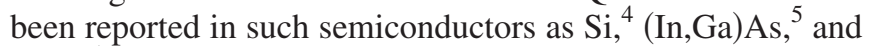
ZnSe. ${ }^{1}$ Thus, the realization of $\mathrm{ZnO}$ QDs in the VolmerWeber growth mode as reported here can be expected to open a feasible avenue to study and create new forms of this material.
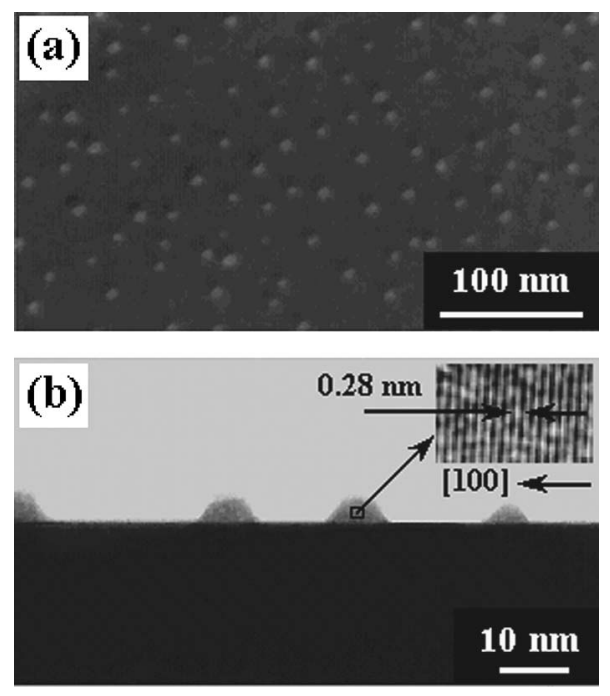

FIG. 2. (a) Plan-view and (b) cross-sectional TEM images of as-prepared $\mathrm{ZnO}$ QDs. An inset high-resolution TEM image shows that the ZnO QDs are single crystal. The growth time is 2 min. studied in this work are open structures and the QD surfaces
Downloaded 31 May 2007 to 130.54 .110 .22 . Redistribution subject to AIP license or copyright, see http:/apl.aip.org/apl/copyright.jsp

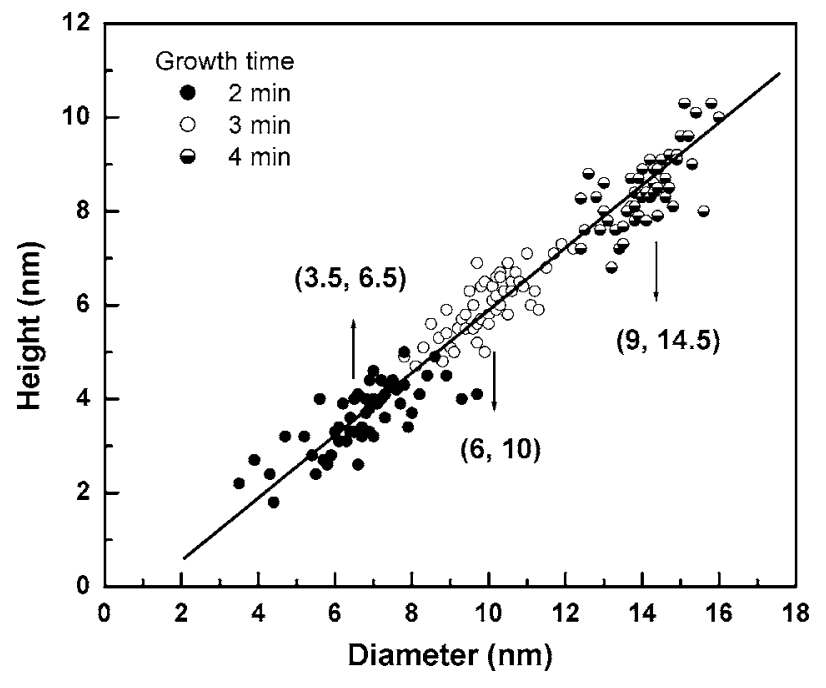

FIG. 3. Dot dimension distribution of individual ZnO QDs determined by TEM measurements. The solid line is the slope obtained by the linear fitting of all the points. The parentheses indicate (average height, average diameter) in units of nanometers.

The dimension distribution of individual QDs deduced from TEM measurements is plotted in Fig. 3. It is clear that the nanodot size becomes larger by the increase of the $\mathrm{ZnO}$ deposition, which is in accordance with the SEM observations. The nanodot dimensions increase from $(3.5,6.5)$ to $(6$, 10) to $(9,14.5)$ as the growth time is up from 2 to 3 to $4 \mathrm{~min}$ if the parentheses indicate (average height, average diameter) in units of nanometers. Although the average size of the dots varies with growth conditions, the distribution of sizes in any one growth is relatively narrow. The dot profile exhibits that the nanodot height is proportional to the diameter, with the height-to-diameter ratio consistently at about 1:1.8, independent of dot size, which is similar to that observed in III-V dots. ${ }^{6} \mathrm{ZnO}$ QDs obtained here are single crystal in nature as illustrated by the high-resolution TEM image in the inset of Fig. 2(b).

ZnO QDs smaller than about $7 \mathrm{~nm}$ in dimension exhibit a so-called quantum confinement effect, an enlargement of the band gap that is typically measured by the blueshift of light absorption. Figure 4 shows the normalized optical absorption spectra of the as-prepared $\mathrm{ZnO}$ QDs. The band gap energies thus determined from absorption spectra reveal the blueshift from 3.34 to 3.41 to $3.57 \mathrm{eV}$ as the QD size decreases. Theoretically, the band gap of $\mathrm{ZnO}$ QDs can also be calculated from nanodot sizes based on the effective mass approximation. ${ }^{15}$ By using the expression developed in Ref. 14 , the calculated values are 3.36, 3.42, and $3.62 \mathrm{eV}$ for $\mathrm{ZnO}$ QDs with the dimensions of $(3.5,6.5),(6,10)$, and $(9,14.5)$, respectively. The theoretical values are consistent with the experimental data, but always with a deviation towards larger values, which is believed to be the results of the use of an infinitely high barrier potential with vanishing wave functions at the boundaries of the equation. Regardless of the discrepancy, the results firmly demonstrate that the band gap of $\mathrm{ZnO}$ QDs shifts to higher energies as the nanodot size decreases. Nanocrystals larger than $7 \mathrm{~nm}$ in height do not show any blueshift and have a band gap close to that of bulk $\mathrm{ZnO}(3.37 \mathrm{eV})$. On the contrary, $\mathrm{ZnO}$ QDs with the dimension of $(3.5,6.5)$ show an energy $\sim 3.57 \mathrm{eV}$, much larger than the band gap of bulk $\mathrm{ZnO}$. Since the QD structures studied in this work are open structures and the QD surfaces 


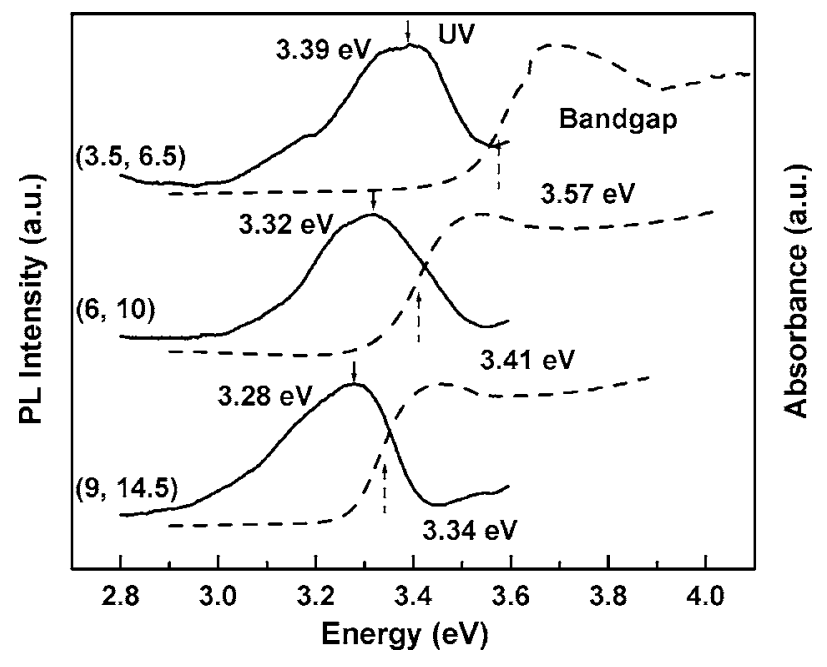

FIG. 4. Room-temperature optical absorption (dashed line) and PL (solid line) spectra of as-prepared $\mathrm{ZnO}$ QDs. The dashed arrows show the band gap of $\mathrm{ZnO}$ QDs determined by the derivative of absorption curves. The solid arrows show the UV emission from $\mathrm{ZnO}$ QDs. The parentheses indicate the nanodot dimensions.

are under the stress free condition, the strain-induced band gap shift should not be taken into account. Accordingly, it is very reasonable to attribute this blueshift of the band gap of $\mathrm{ZnO}$ QDs with increasing dot sizes due to the quantum confinement effects.

Figure 4 also shows the normalized global roomtemperature PL spectra of the as-prepared ZnO QDs with different average sizes. The ultraviolet (UV) emission occurs in $\mathrm{ZnO} \mathrm{QDs}$ and shifts to higher energies (from 3.28 to 3.32 to $3.39 \mathrm{eV}$ ) as the nanodot dimension decreases. This blueshift behavior of PL peak position is naturally explained by the expected effect of quantum confinement as discussed above. In general, quantum confinement shifts the energy levels of the conduction and valence bands apart, giving rise to a blueshift in the transition energy as the nanodot size decreases. The UV luminescence is believed to come from bound or localized excitons in $\mathrm{ZnO}$ QDs. ${ }^{16}$ It is well known that the exciton localization is so strong in $\mathrm{ZnO}$ QDs that it can manifest itself even at room temperature. ${ }^{16,17}$ As clearly identified in Fig. 4, by varying the nanodot size, the room-temperature luminescence in the UV region can be readily tuned from 3.28 to $3.39 \mathrm{eV}$ for $\mathrm{ZnO}$ QDs obtained here. This size-dependent emission wavelength manipulation indicates that this material system may be an ideal candidate for use in tunable emitters for biomedical labels, light emitting diodes (LEDs), lasers diodes (LDs), and sensors.

In Fig. 4 the Stokes shift can be observed as an indicator of the exciton localization in $\mathrm{ZnO}$ QDs. The Stokes shift becomes larger (from 60 to 90 to $180 \mathrm{meV}$ ) as the nanodot size decreases, suggesting the enhancement of the degree of exciton localization. The exciton localization can effectively annihilate the nonradiative recombination centers, thereby having a significant effect on the device performance such as determining the emission energy in LEDs and contributing to luminescent efficiency and optical gain within laser structures. ${ }^{16,17}$ In addition, the dead layer could be formed near the QD surface with the thickness up to $1.6 \mathrm{~nm}$ due to the specific dielectric constants and effective masses of hole and electron in $\mathrm{ZnO}^{18}$ The rather thick dead layer in $\mathrm{ZnO}$
QDs is expected to further improve luminescence owing to the exciton separation from the surface defects. These characteristics make $\mathrm{ZnO}$ QDs very promising for optoelectronic applications.

Previous studies on $\mathrm{CdSe}$ and $\mathrm{ZnSe}$ QDs revealed that they could be unstable in some cases. ${ }^{2}$ To clarify the stability of $\mathrm{ZnO}$ QDs, we evaluated them at intervals after deposition. No change in the SEM images was observed for all the samples, and moreover there was almost no difference appeared in the optical absorption and PL spectra, both position and shape, even after being aged over 15 months. The samples were kept in an ordinary silica-gel desiccator under atmospheric conditions. These observations indicate that the $\mathrm{ZnO}$ nanodot structures and their characteristics are time independent; that is to say, $\mathrm{ZnO}$ QDs are very stable as expected. The results presented here strongly suggest that $\mathrm{ZnO}$ QDs should be more suitable for the practical shortwavelength applications as compared with $\mathrm{CdSe}$ and $\mathrm{ZnSe}$ QDs.

In summary, we have realized self-assembled $\mathrm{ZnO}$ QDs on solid substrates by a VPT process in the Volmer-Weber growth mode. The nanodot sizes could be readily controlled by varying the growth durations. The color-tunable nature of QDs was unambiguously demonstrated by optical absorption and PL spectra in the $\mathrm{ZnO}$ system. The obtained ZnO QDs were of high quality and stability in their features. The recognition presented in this work opens the door for the creation, study, and application of ZnO QDs in a systematic and controlled manner.

This work was supported by the Key Project of National Natural Science Foundation of China under Grant No. 50532060 .

${ }^{1}$ M. C. H. Liao, Y. H. Chang, Y. F. Chen, J. W. Hsu, J. M. Lin, and W. C. Chou, Appl. Phys. Lett. 70, 2256 (1997).

${ }^{2}$ I. Suemune, T. Tawara, T. Saitoh, and K. Uesugi, Appl. Phys. Lett. 71, 3886 (1997).

${ }^{3}$ N. Myung, Y. Bae, and A. J. Bard, Nano Lett. 3, 1053 (2003).

${ }^{4}$ A. Malachias, R. Magalhães-Paniago, S. Kycia, and D. G. Cahill, J. Appl. Phys. 96, 3234 (2004).

${ }^{5}$ A. F. Tsatsul'nikov, A. R. Kovsh, A. E. Zhukov, Y. M. Shernyakov, Y. G. Musikhin, V. M. Ustinov, N. A. Bert, P. S. Kop'ev, Z. I. Alferov, A. M. Mintairov, J. L. Merz, N. N. Ledentsov, and D. Bimberg, J. Appl. Phys. 88, 6272 (2000).

${ }^{6}$ S. Fafard, D. Leonard, J. L. Merz, and P. M. Petroff, Appl. Phys. Lett. 65, 1388 (1994).

${ }^{7}$ Z. K. Tang, G. K. L. Wong, P. Yu, M. Kawasaki, A. Ohtomo, H. Koinuma, and Y. Segawa, Appl. Phys. Lett. 72, 3270 (1998).

${ }^{8}$ Z. L. Wang, J. Phys.: Condens. Matter 16, R829 (2004).

${ }^{9}$ E. M. Wong, J. E. Bonevich, and P. C. Searson, J. Phys. Chem. B 102, 7770 (1998).

${ }^{10}$ L. Guo, S. Yang, C. Yang, P. Yu, J. Wang, W. Ge, and G. K. L. Wong, Appl. Phys. Lett. 76, 2901 (2000).

${ }^{11}$ C. L. Yang, J. N. Wang, W. K. Ge, L. Guo, S. H. Yang, and D. Z. Shen, J. Appl. Phys. 90, 4489 (2001).

${ }^{12}$ A. L. Roest, A. Germeau, J. J. Kelly, D. Vanmaekelbergh, G. Allan, and E. A. Meulenkamp, ChemPhysChem 4, 959 (2003).

${ }^{13}$ S. W. Kim, M. Ueda, M. Funato, Sg. Fujita, and Sz. Fujita, J. Appl. Phys. 97, 104316 (2005).

${ }^{14}$ J. G. Lu, Z. Z. Ye, J. Y. Huang, L. P. Zhu, B. H. Zhao, Z. L. Wang, and Sz. Fujita, Appl. Phys. Lett. 88, 063110 (2006).

${ }^{15}$ Y. Kayanuma, Phys. Rev. B 38, 9797 (1988).

${ }^{16}$ V. A. Fonoberov and A. A. Balandin, Appl. Phys. Lett. 85, 5971 (2004).

${ }^{17}$ V. A. Fonoberov and A. A. Balandin, Appl. Phys. Lett. 86, 226101 (2005).

${ }^{18}$ V. A. Fonoberov and A. A. Balandin, Phys. Rev. B 70, 195410 (2004). 